\title{
Thoracoscopic lobectomy in a lung cancer patient with severe hemophilia: A case report
}

\author{
XING-YU LIN, ZHI-GUANG YANG, PENG ZHANG, YUN-PENG LIU, \\ CHENG-XIANG WANG and GUO-GUANG SHAO
}

\author{
Department of Thoracic Surgery, First Hospital of Jilin University, Changchun, Jilin 130021, P.R. China
}

Received October 21, 2014; Accepted July 21, 2015

DOI: $10.3892 / \mathrm{ol} .2015 .3717$

\begin{abstract}
Hemophilia is a hereditary disease with impaired blood coagulation due to a genetic deficiency of blood coagulation factors. Hemophilia often causes spontaneous life-threatening bleeding, so patients with hemophilia are often not suitable for any surgery that may cause iatrogenic bleeding and threaten the life of the patient. Therefore, surgery in lung cancer patients with hemophilia is extremely rare. The present study reported the case of a lung cancer patient with hemophilia who presented with a persistent cough. A mass was revealed by computed tomography and the patient underwent a successful thoracoscopic right lower lobectomy. The study discusses the patient's diagnosis and treatment options for hemophilia A and lung cancer, including indications for thoracoscopic lobectomy, pre-operative preparation and post-operative care, and other treatment options are discussed. The literature is also reviewed on this subject.
\end{abstract}

\section{Introduction}

Lung cancer is one of the most prevalent health problems, accounting for $>1.6$ million new cancer cases and 1.4 million cancer-related fatalities each year worldwide (1). The survival of lung cancer patients, particularly for non-small cell lung cancer (NSCLC), which constitutes $\sim 80 \%$ of all lung cases diagnosed, mostly relies on early detection. Surgery is the most critical treatment option to improve the survival of NSCLC patients, however, only those patients with early-stage disease and those who are physically able can undergo surgical procedures. Hemophilia is an inherited blood coagulation disorder caused by a genetic deficiency of blood coagulation factors. Hemophilia often causes life-threatening bleeding (2). The

Correspondence to: Dr Guo-Guang Shao, Department of Thoracic Surgery, First Hospital of Jilin University, 71 Xinmin Avenue, Changchun, Jilin 130021, P.R. China

E-mail: guoguangshao@sohu.com

Key words: lung cancer, hemophilia, thoracoscopy, coagulation factor VIII complication of hemophilia often presents with deep internal bleeding, hemophilic arthropathy and transfusion-transmitted infection (3). Patients with hemophilia are often not suitable for any surgery, which may cause iatrogenic bleeding and be life-threatening. The present study report the unusual case of a lung cancer patient with hemophilia A who underwent surgery successfully. The patient's diagnosis, treatment options for hemophilia A and lung cancer, such as indications for thoracoscopic lobectomy, pre-operative preparations and post-operative cares, and other treatment options are discussed. Written informed consent was obtained from the patient. The literature is also reviewed for the treatment and management of such patients.

\section{Case report}

Admission to hospital. In June 2013, a 53-year-old male patient seeking medical attention due to a cough with sputum that had persisted for two month presented to the First Hospital of Jilin University (Changchun, Jilin, China). The patient underwent a computed tomography (CT) scan, which showed a $3.0 \times 2.0-\mathrm{cm}$ mass in the right lower lobe of the lung and was subsequently admitted. A physical examination showed stable vital signs, stained body skin, stained conjunctival and oral mucosa, swollen joints, and darker old scars on the forehead. The patient could not walk independently. Moreover, the bilateral thoracic size was symmetrical, bilateral breathing was smooth and bilateral fremitus was normal. Auscultation of the lungs revealed slightly weaker breathing sounds in the right lower lung. The systemic lymph nodes were normal. Auxiliary lung CT showed a 3.0x2.0-cm mass in the right lower lobe with irregular edges and burrs, but there was no significant mediastinal lymph node enlargement. Positron emission tomography-computed tomography (Fig. 1) in the right lower lobe lung revealed a high metabolic mass with possible large lumps, $3.0 \times 2.0 \mathrm{~cm}$ in size. Cardiac function was rated with a fractional shortening value of $32 \%$ (normal range, $25-35 \%$ ) and an ejection fraction value of $68 \%$ (normal range, $>50 \%$ ), while lung function was recorded with a $2.86 \mathrm{l} / \mathrm{sec}$ forced expiratory volume in one second (FEV1; normal range, $80-120 \mathrm{l} / \mathrm{sec}$ ). There was no significant mediastinal lymph node metastasis. Laboratory tests showed an activated partial thromboplastin time (APTT) of $66.5 \mathrm{sec}$ (normal range, 20-40 sec), a prothrombin time of $16.3 \mathrm{sec}$ 
Table I. Dynamic monitoring of the patient for continued factor VIII infusion therapy.

\begin{tabular}{crcc}
\hline Days post-surgery & Dose of factor VIII, IU & Blood levels of factor VIII, $\%$ & Chest fluid volume and color trait \\
\hline 1 & 3500 & 120.00 & 600 ml of dark red bloody fluid \\
2 & 3500 & 128.10 & 250 ml of dark red bloody fluid \\
3 & 3500 & 133.50 & 150 ml of pinkish transparent liquid \\
4 & 3500 & 207.50 & 140 ml of pinkish transparent liquid \\
5 & 3500 & 160.20 & 100 ml of pinkish transparent liquid \\
6 & 3500 & 140.20 & Pleural drainage tube removed \\
7 & 2000 & 61.00 & - \\
9 & 2000 & 111.00 & - \\
11 & 1000 & 57.00 & - \\
13 & 500 & 22.00 & - \\
15 & 0 & - & Discharged from hospital \\
\hline
\end{tabular}
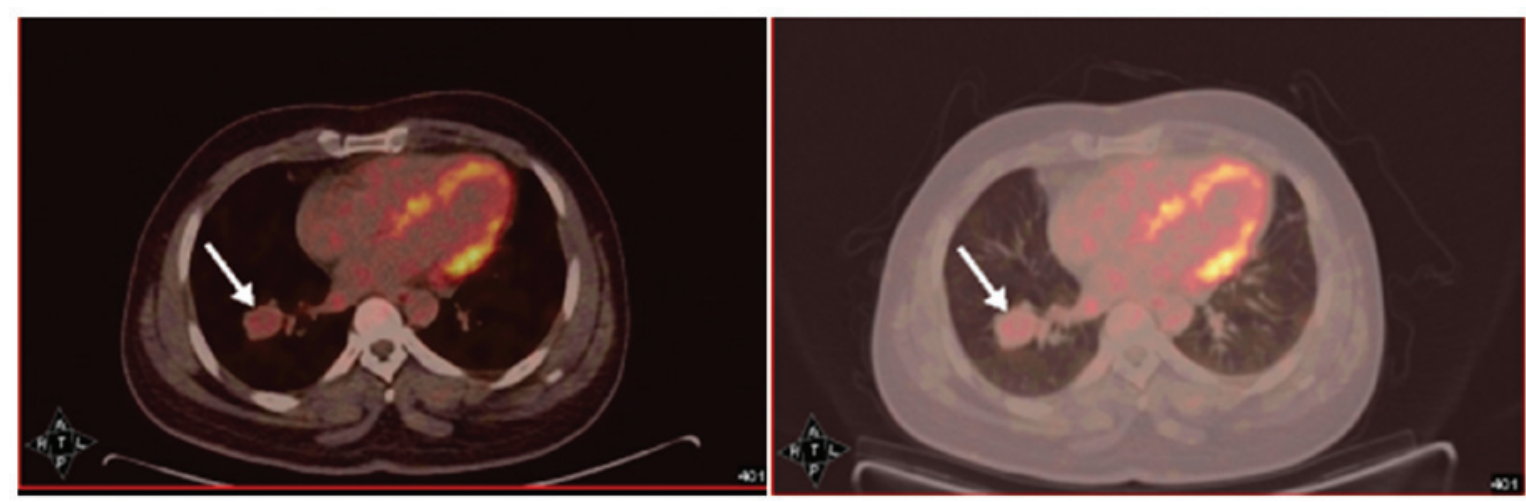

Figure 1. Positron emission tomography-computed tomography showing a 3.0x2.0-cm mass (arrow) in the right lower lobe of the lung.

(normal range, 9-13 sec), a fibrinogen (Fbg) level of $6.34 \mathrm{~g} / 1$ (normal range, $2-4 \mathrm{~g} / \mathrm{l}$ ), a platelet level of $323 \times 10^{9} / 1$ (normal range, $\left.125-250 \times 10^{9} / 1\right)$, an alanine aminotransferase level of $23 \mathrm{U} / 1$ (normal range, 10-60 U/1) and 0.80\% blood coagulation factor VIII (normal range, 50-150\%). It was noted that 40 years ago, the patient had suffered excessive bleeding after a minor trauma and was subsequently diagnosed with influenza with severe hereditary hemophilia and underwent a long-term treatment with factor VIII (monthly infusion of $\sim 250 \mathrm{mg}$ ), and that the bleeding symptoms were controlled well.

Pre-surgical preparations and surgery. The patient underwent a routine health examination, which revealed a normal electrocardiogram, normal echocardiography and 90\% FEV1/forced vital capacity. The family members and patient refused a bronchoscopy. The patient was subjected to dynamic monitoring of clotting factor VIII levels, with timely infusion of clotting factor VIII (Bai Keqi, Bayer HealthCare AG, Beijing, China) according to the formula: Required factor VIII units (IU) / Ci $=0.5 \mathrm{x}$ patient body weight $(\mathrm{kg}) \mathrm{x}$ required elevated levels of factor VIII activity (\% of normal). This resulted in administration of $\sim 3,400$ IU daily, and 1 day prior to surgery, the levels of factor VIII had reached $87 \%$. During surgery, the patient was infused with 10 units of red blood cell suspension and 1,000 $\mathrm{ml}$ plasma.
Surgical anesthesia was used for the thoracoscopic right lower lobe resection and lymph node dissection. During and after the surgery, the patient did not experience any significant bleeding $(\sim 400 \mathrm{ml})$, and was subsequently infused with two units of red blood cells and $400 \mathrm{ml}$ plasma.

Post-surgical care. Following the successful surgical removal of the lung cancer lesion, the patient continued to receive factor VIII infusion therapy and dynamic monitoring (Table I).

The patient also underwent conventional post-operative treatment with ambroxol (60 mg, twice daily for 7 days), omeprazole (40 mg, twice daily for 2 days) and cefoxitin sodium (2,000 $\mathrm{mg}$, twice daily for 5 days). At $24 \mathrm{~h}$ post-surgery, the patient was generally in good condition, exhibiting stable vital signs and weak breathing sounds on lung auscultation. The number and percentage of white blood cells gradually reached normal levels. Routine coagulation tests showed an APTT of $44.4 \mathrm{sec}$ and an Fbg level of $4.82 \mathrm{~g} / \mathrm{l}$. Post-operative thoracic drainage gradually decreased, the coloration changing from dark red bloody fluid into pink transparent liquid. The patient had generally recovered 4 days later, with stable vital signs and lung CT showing post-operative changes in lung compliance, with good lung re-expansion, although there was still a small liquid shadow in the right lower lobe. Blood and liver functions were normal. On post-operative day 6 , the chest tube was removed and the patient showed no evident discomfort. 
On pathological examination, a $2.3 \times 3.1 \times 2.2 \mathrm{~cm}$ tumor was identified in the right lower lobe. The tumor was unencaspulated, grey and yellow in colour, and composed of poorly differentiated cells with unclear cytoplasmic boundaries and round nuclei. A pathological diagnosis of a right lower lobe low-grade neuroendocrine tumor (formerly carcinoid tumor) [TNM stage, T2aN0M0 (4)] without lymph node metastasis was made. On post-operative day 15 , the patient was discharged.

\section{Discussion}

The lifespan of patients with severe hemophilia who do not receive an adequate modern treatment regimen is markedly reduced. Indeed, these patients often do not reach maturity. By contrast, patients with hemophilia who receive appropriate therapy go on to live 50-60 years (5). Such patients, however, may suffer from other diseases, including lung cancer. As surgery may cause uncontrolled bleeding in these patients, they are often not suitable for surgery.

In the present case study, the case of a hemophilia patient with lung cancer who underwent surgical removal of a tumor lesion is presented. Indeed, to date, there have been few studies describing minor surgical procedures in hemophilia patients (6-8), however, no cases of lobectomy use for the removal of a lung tumor have been reported. As these patients lack the hemophilia clotting factor due to a genetic defect, such surgical procedures are believed to be extremely risky. Thus, extensive pre-operative preparations and post-operative care are of utmost importance for these patients. Through the course of the diagnosis and treatment in the present case, the maintenance of factor VIII levels was found to be critical, since the biological half-life of factor VIII is between 6 and $14 \mathrm{~h}$. As such, regular monitoring of blood clotting factor levels during treatment adjustment of infusion doses and intervals is crucial (3). Bai Keqi factor VIII is a recombinant preparation for the treatment of hemophilia factor, with better efficacy and safety profiles when compared with plasma or cryoprecipitate direct input treatment, which are considered the conventional preoperative treatments for patients with hemophilia. However, recombinant factor VIII preparation for Chinese patients has not yet been reported. Therefore, the selection and use of Bai Keqi clotting factor drug was a better choice and following such treatment, patients have exhibited no symptoms of factor VIII-specific antibody generation, which is a specific neutralization reaction against such treatment in the host (9). The present patient responded well to treatment, and 5 days after surgery, the post-operative drainage tubes were removed and the drug dose was reduced to half and a normal blood factor VIII level (>80\%) was maintained, although blood factor VIII levels in the patient declined. Specifically, the patient did not show any evident signs of discomfort or adverse symptoms, the drainage liquid color gradually cleared from dark red to yellow and transparent, and the post-operative thoracic drainage was gradually reduced. Thus, the treatment was considered successful.

Lung cancer surgery or thoracoscopic thoracic surgery could be an important option for such patients, if they are carefully treated. The present study indicates that the treatment course, and drug choice and amount are crucial factors for successfully managing these patients prior to, during and following surgical procedures. In the present study, the choice of surgery was a minimally invasive thoracoscopic technique. For post-operative care, the coagulation factor, and pleural drainage volume and color were dynamically monitored, which was consistent with and supported by other studies $(10,11)$.

In the current study, the case of a lung cancer patient with hemophilia who underwent successful surgical resection of a tumor lesion was presented. Hemophilia can lead to spontaneous hemorrhage and coagulopathy and thus, hemophilia patients exhibit a high risk when undergoing surgery. However, minimally invasive surgical techniques and genetically engineered drug treatments have been developed which decrease this risk. The patient in the present study underwent a thoracoscopic lobectomy following the administration of a pre-operative preparation and the procedure was successful. Future studies of such patients will improve knowledge with regard to the clinical management of hemophilia patients that require surgery for the treatment of other conditions.

\section{References}

1. Rakovich G and Tremblay L: Tailored therapy in lung cancer. Can Respir J 20: 367-368, 2013.

2. Chen HZ: Practical Internal Medicine. 10th edition. People's Medical Publishing House, Beijing, 1998.

3. Siboni SM, Biguzzi E, Solimeno LP, Pasta G, Mistretta C, Mannucci PM and Peyvandi F: Orthopaedic surgery in patients with von Willebrand disease. Haemophilia 20: 133-140, 2014.

4. Ettinger DS, Wood DE, Akerley W, et al: Non-small cell lung cancer, version 1.2015. J Natl Compr Canc Netw 12: 1738-1761, 2014.

5. Chorba TL, Holman RC, Strine TW, Clarke MJ and Evatt BL: Changes in longevity and causes of death among persons with hemophilia A. Am J Hematol 45: 112-121, 1994.

6. Washino S, Hirai M, Kobayashi Y, Saito K and Miyagawa T: Heavy hematuria requiring cystectomy in a patient with hemophilia A: A case report and literature review. BMC Urol 15: 84, 2015.

7. Ingram GI: Haemophilia and the forbidden abdomen. Haemophilia 6: 719-722, 2000.

8. Lavery HJ, Senaratne P, Gainsburg DM and Samadi DB: Robotic prostatectomy in a patient with hemophilia. JSLS 14: 439-441, 2010.

9. Beutler E, Lichtman MA, Coller BS, Kipps TJ and Seligsohn U: Williams Hematology. 6th edition. McGraw-Hill Professional, New York, 2001.

10. Demmy TL and Nwogu C: Is video-assisted thoracic surgery lobectomy better? Quality of life considerations. Ann Thorac Surg 85: S719-S728, 2008.

11. Craig SR, Leaver HA, Yap PL, Pugh GC and Walker WS: Acute phase responses following minimal access and conventional thoracic surgery. Eur J Cardiothorac Surg 20: 455-463, 2001. 